\section{Armonización iberoamericana de los instrumentos PCAT para la evaluación del primer nivel de atención}

\author{
Jacqueline Ponzo, ${ }^{1}$ \\ Silvina Berra, ${ }^{2}$ \\ Alexandra Giraldo-Osorio, ${ }^{3}$ \\ M. Isabel Pasarín, ${ }^{4}$ \\ Christine Leyns, ${ }^{5}$ \\ Consuelo Vélez Álvarez, ${ }^{3}$ \\ Carlos Soto, ${ }^{6}$ Diana Molina, ${ }^{7}$ \\ Alejandra Fuentes-García, ${ }^{8}$ \\ Lisiane Hauser, ${ }^{9}$ \\ Jenny Magne, ${ }^{5}$ \\ Victor Chicaiza, ${ }^{7}$ \\ Teresa Torres, ${ }^{6}$ \\ Franco González Mora, ${ }^{2}$ \\ Agustina Terra, ${ }^{2}$ \\ Erno Harzheim, ${ }^{10}$ \\ Patricia Ferre, ${ }^{11}$ \\ Victor Borja- Aburto, ${ }^{6}$ \\ Mauricio Fuentes ${ }^{8}$
}

Forma de citar Ponzo J, Berra S, Giraldo-
Osorio A, Pasarín MI, Leyns C, Vélez-Álvarez
C et al. Armonización iberoamericana de
los instrumentos PCAT para la evaluación
del primer nivel de atención. Rev Panam
Salud Publica. 2018;42:e80. https://doi.org/
10.26633/RPSP.2018.80

\section{Estimado editor,}

Cuarenta años después de Alma Ata, el mundo está reforzando la consciencia sobre la importancia de los atributos y funciones del primer nivel de atención, pero aún queda mucho por hacer para implementarlos, medirlos y aprender de las experiencias exitosas de algunos países. El conjunto de instrumentos Primary Care Assessment Tools (PCAT), desarrollados en los años 90 en Estados Unidos de América por Starfield y Shi (1), se ha mostrado como la herramienta más completa para el análisis de la atención primaria de la salud (APS) y su utilidad fue reconocida en numerosos países y regiones del mundo.

El objetivo de esta carta es comunicar preliminarmente el proceso de armonización de las versiones PCAT ya adaptadas en diversos países de Iberoamérica y subrayar la importancia de estas herramientas para evaluar y comparar el primer nivel de atención, particularmente en sistemas de salud fundados en la estrategia de APS.

En Iberoamérica, desde 2005 se realizaron procesos de adaptación transcultural en España (2) y Brasil (3), seguidos de Argentina (4) y Uruguay (5), supervisados por la Prof. Starfield. Después, otros cinco países condujeron procesos similares con colaboración de al menos dos investigadores de los cuatro primeros países. En todos los casos se siguió un método riguroso de adaptación transcultural, por lo que los instrumentos de los nueve países tenían un alto grado de equivalencia. A su vez, en los sucesivos procesos de adaptación aparecieron diferencias debidas a necesidades emergentes de la realidad local y se identificaron necesidades de actualización, ratificadas oportunamente por los autores (Starfield B, 2010, comunicación personal; Shi L, 2017, reunión en Vancouver, Canadá). Los fuertes vínculos entre los países que desde 2010 conforman la Colaboración Iberoamericana IAPCAT propiciaron un proceso de armonización y actualización que se ejecutó durante 2016 y 2017.

El trabajo se dirigió a armonizar los enunciados y adecuar el contenido de los instrumentos al contexto cultural, sanitario y epidemiológico latinoamericano, considerando la evidencia científica actual y el desarrollo tecnológico en los procesos de atención, manteniendo los principios teóricos y operativos del modelo original, mediante una perspectiva regional que capitalizara los avances logrados en cada país. Participaron en este proyecto 19 investigadores de 10 grupos de 9 países: Argentina, Bolivia, Brasil, Colombia, Chile, Ecuador, España, México y Uruguay. Se ejecutaron cuatro etapas de trabajo: 1) Conformación del fondo de versiones PCAT disponibles en Iberoamérica de las cuatro ediciones de PCAT (PE-proveedores, FEgestores o equipos de salud, AE-usuarios adultos y CE-usuarios niños y adolescentes), incluyendo la original de los Estados Unidos. 2) Identificación de enunciados predominantes de cada ítem y evaluación de su adecuación cultural en cada país. 3) Valoración de la equivalencia de ítems similares entre las diferentes ediciones de PCAT (PE, FE, AE y CE) de todos los países para cada ítem. 4) Focalización del trabajo en ítems con problemas de equivalencia o de actualidad. Las deliberaciones se realizaron por conferencia sincrónica de todo el panel de expertos o de subgrupos de trabajo, vía WebEx o Skype, tras lo cual se elaboraron minutas que fueron revisadas y corregidas por todos los participantes. Se trabajó con 26 cuestionarios PCAT de Iberoamérica (8 PE, $5 \mathrm{FE}, 8 \mathrm{AE}, 5 \mathrm{CE}$ ), además de las 4 versiones originales. Se revisaron, compararon y valoraron 1311 ítems de la edición PE, 865 ítems de la edición FE, 946 ítems de la edición AE y 472 ítems de la edición CE. Las versiones preliminares obtenidas hasta el momento serán sometidas a pruebas de comprensión, fiabilidad y validez.

Este es un artículo de acceso abierto distribuido bajo los términos de la licencia Creative Commons Attribution-NonCommercial-NoDerivs 3.0 IGO, que permite su uso, distribución y reproducción en cualquier medio, siempre que el trabajo original se cite de la manera adecuada. No se permiten modificaciones a los artículos ni su uso comercial. Al reproducir un artículo no debe haber ningún indicio de que la OPS o el artículo avalan una organización o un producto específico. El uso del logo de la OPS no está permitido. Esta leyenda debe conservarse, junto con la URL original del artículo. 
1 Universidad de la República, Facultad de Medicina, Montevideo, Uruguay

2 Universidad Nacional de Córdoba, Escuela de Salud Pública, Córdoba, Argentina

3 Universidad de Caldas, Manizales, Colombia

4 Agència de Salut Pública de Barcelona, Barcelona, España

5 Asociación Interdisciplinaria de Atención Primaria de Salud, Bolivia

6 Instituto Mexicano del Seguro Social, Ciudad de México, México

Ministerio de Salud Pública, Quito, Ecuador

8 Universidad de Chile, Escuela de Salud Pública, Santiago Chile, Chile

9 Universidade Federal do Rio Grande do Sul. Faculdade de Medicina. Programa de PosGraduação em Epidemiologia, Porto Alegre, RS, Brasil

10 Secretaria Municipal de Saúde de Porto Alegre, Porto Alegre, RS. Brasil

11 Escuela Andaluza de Salud Pública, Granada, España
La estructura de trabajo en red, con soporte en los recursos institucionales disponibles, permitió sostener este proceso de investigación y desarrollo propiciado por la motivación de los investigadores locales. Este proceso dio valor, sistematizó y capitalizó la experiencia acumulada en la región, las revisiones críticas, las actualizaciones y las particularidades culturales de los nueve países participantes. El resultado más tangible son las versiones preliminares del conjunto de instrumentos (ahora denominado IA-PCAT) más adecuados a la región y a la situación epidemiológica y sanitaria actual, sobre la base de los principios, conceptos y constructos originales del modelo PCAT, es decir, la APS. La diversidad geográfica y cultural de los países participantes, que incluye a la Región Andina, el Cono Sur, Mesoamérica y la Península Ibérica, permite presumir que el instrumento cuenta con idoneidad para avanzar en el proceso de prueba de comprensión y aplicación aún en países de la región que no han realizado adaptaciones previas, posibilitando la realización de estudios de evaluación de la APS comparativos en la región a corto plazo. Este trabajo puede ser de interés para otras regiones del mundo donde se utiliza el modelo PCAT y se podría realizar un proceso de armonización similar y una actualización equivalente.

Financiamiento. El proceso de armonización tuvo el apoyo del Departamento de Sistemas y Servicios de Salud de la Organización Panamericana de la Salud (Washington, DC, Estados Unidos) para la contratación de apoyo administrativo. Los integrantes del grupo de trabajo participaron de forma voluntaria y ad-honorem.

Declaración. Las opiniones expresadas en este manuscrito son responsabilidad de los autores y no reflejan necesariamente los criterios ni la política de la RPSP / PAJPH, la OPS y las otras instituciones a las cuales los autores están afiliados.

\section{REFERENCIAS}

1. Starfield B. Primary Care: Balancing Health Needs, Services and Technology. New York: Oxford University Press; 1998.

2. Pasarín MI, Berra S, Rajmil L, Solans M, Borrell C, Starfield B. Un instrumento para la evaluación de la atención primaria de salud desde la perspectiva de la población. Aten Primaria. 2007;39(8):395-401.

3. Harzheim E, Starfield B, Rajmil L, Álvarez-Dardet C, Stein AT. Consistência interna e confiabilidade da versão em português do instrumento de avaliação da atenção primária (PCATool-Brasil) para serviços de saúde infantil. Cad Saude Publica. 2006;22:1649-59.

4. Berra S, Hauser L, Audisio Y, Mántaras J, Nicora V, de Oliveira MMC, Starfield B, Harzheim B. Validez y fiabilidad de la versión argentina del PCAT-AE para evaluar la atención primaria de salud. Rev Panam Salud Pública. 2013;33(1):30-9.

5. Pizzanelli M, Ponzo J, Buglioli M, Toledo A, Casinelli M, Gómez A. Validación de Primary Care Assessment Tool (PCAT) en Uruguay. Rev Med Urug. 2011;27(3):187-9. 